\title{
Effect of Continues Midwifery Support in Labor on the Childbirth Experience and Self-Esteem of Primipara Women: A Randomized Controlled Trial
}

Nasrin Soleimani

Hamadan University of Medical Sciences

Mansoureh Refaei

Hamadan University of Medical Sciences

Farideh Kazemi ( $\sim$ faridehkazemi21@yahoo.com )

Hamadan University of Medical Sciences

\section{Research Article}

Keywords: pregnancy, self-esteem, labor, doula, trial

Posted Date: September 20th, 2021

DOI: https://doi.org/10.21203/rs.3.rs-833826/v1

License: (c) (1) This work is licensed under a Creative Commons Attribution 4.0 International License.

Read Full License 


\section{Abstract}

Background: The World Health Organization states that mothers during labor and delivery should be supported by people with whom they feel comfortable, because one of the non-pharmacological methods of relieving labor pain is the continued support of midwives in labor. The primary objectives of this study were to determine the effect of continuous midwifery support in labor on delivery experience and selfesteem of nulliparous women 6 weeks after delivery.

Methods: This randomized controlled trial was performed in 2021 on 70 pregnant women admitted to the delivery ward who met the inclusion criteria. Participants were randomly allocated to two groups through balanced block randomization with a block size of 4. Participants completed a demographic and midwifery information questionnaire before entering the study. In the intervention group, in addition to routine labor care, the researcher was present at the mother's bedside and was present with the mother continuously throughout the labor and up to 2 hours after delivery and provided the necessary support. The control group received only routine labor and postpartum care. Six weeks after delivery, participants in both groups were contacted to complete the CEQ and Rosenberg self-esteem questionnaires. Finally, data were collected and analyzed. Significance level was considered 0.05 .

Results: By controlling the effect of duration of the first and second stages of labor as potential confounders, the mean (sd) of childbirth experience 6 weeks after delivery was 70.92 (7.07) in the intervention group and 59.69 (7.08) in the control group, and this difference was statistically significant $(p<0.001)$. The amount of effect size indicates a very strong effect of the intervention on improving the childbirth experience (Cohen's $d=1.69 ; 95 \% \mathrm{Cl}$ : 1.12, 2.26). Comparison of the mean of maternal selfesteem showed that this rate in the intervention group was 2 scores higher than the control group and this difference was statistically significant $(p=0.05)$. Cohen's $d$ indicates the average effect of intervention on mothers' self-esteem score (Cohen's $d=0.56 ; 95 \% \mathrm{Cl}: 0.06,1.05$ ).

Conclusions: Continuous support of the midwife in labor and up to 2 hours after delivery can lead to a better childbirth experience and increase the mother's self-esteem up to 6 weeks after delivery.

Trial registration: IRCT ID: IRCT20201102049233N1, First Registration date: 2021-06-04

\section{Background}

Childbirth is described as a multidimensional experience that includes mental, emotional and physical factors (1). The importance of the experience of childbirth is so great that the World Health Organization (WHO), while emphasizing maternal and child health, has put the provision of integrated services for maternal mental health on the agenda of development goals for the years after 2015 (2). A positive childbirth experience can improve the mother's well-being and the relationship between the baby and the mother (3), and negative childbirth experiences can increase the risk of postpartum depression and by increasing the fear of childbirth and creating a negative attitude towards pregnancy, it may lead to a 
reluctance to have children in the future, choosing a cesarean section after a previous vaginal delivery, and poor breastfeeding $(4,5)$.

In past decades and in different cultures, women were supported by other women during labor and delivery. But since the middle of the twentieth century, the majority of women in many countries have given birth in hospitals instead of at home, and this has made continued support in labor a non-routine activity (6). The companion acts as a supporter of the woman, helps the mother to communicate with the treatment staff and express her preferences, and provides encouragement, reassurance, and physical comfort to the mother (7). Health care professionals emphasize respect, reverence, and emotional support during childbirth (8). WHO states that mothers during labor and delivery should be supported by people with whom they feel comfortable, because one of the non-pharmacological methods of relieving labor pain is the continued support of midwives in labor. In developed countries, in $95 \%$ of cases, childbirth is supported by a spouse or other family member. Having a trained midwife with the mother in the delivery room helps reduce anxiety and improve labor (9). Continuous support during labor reduces medical interventions such as oxytocin use, analgesia and delivery with tools and complications such as postpartum depression, failure to breastfeed and anxiety (10), and improves women's experience of childbirth (11). In addition, midwifery care during labor and involving mothers in decision-making during childbirth increases their self-confidence and self-esteem $(12,13)$.

According to the authors of this article, few studies have been conducted on the effect of continued midwifery support in labor on delivery experience and women's self-esteem, and the implications in various studies have focused on the short-term effects of support on labor duration, maternal and fetal outcomes, the rate of spontaneous vaginal delivery or cesarean section, and instrumental deliveries ( 6 , 13-15). In a systematic review published in Cochrane, the authors suggested that because short-term outcomes were considered in the studies included in this review, studies should be conducted on longterm outcomes and in low-income countries (6). Therefore, the primary objectives of this study were to determine the effect of continuous midwifery support in labor on the childbirth experience and selfesteem of nulliparous women 6 weeks after delivery.

\section{Method}

The sample size was calculated with Stata-13 software, using the data obtained from the study of Lathrop et al. $(16)(\mathrm{m} 1=13.0, \mathrm{~m} 2=13.75, \mathrm{sd} 1=1.24, \mathrm{sd} 2=0.81)$ and $\mathrm{a}=0.05$ and power $=0.80$ and with $10 \%$ loss.

Inclusion criteria were age 18-35 years, no history of childbirth, live fetus with cephalic presentation, singleton pregnancy, gestational age 37 to 41 weeks, not having pregnancy complications (gestational diabetes, gestational hypertension, bleeding, placental abruption and placenta previa) or chronic medical problems (such as diabetes, high blood pressure, heart, kidney problems, etc.), dilatation of $4-5 \mathrm{~cm}$ at the time of hospitalization, literacy, no history of infertility, and not having a companion midwife or trained companion. The exclusion criterion was the participant's refusal to continue participating in the research. 
The questionnaires used in this study included demographic and obestetric information questionnaire (including age, education, occupation, gestational age, marital satisfaction, household income, and pregnancy status), labor and delivery status questionnaire (including the duration of the first and second stages of labor, the baby's weight, the state of oxytocin intake, how the placenta came out, the person performing the delivery, and the sex of the baby), childbirth experience questionnaire (CEQ) and Rosenberg self-esteem scale.

The CEQ was designed by Dencker et al. and consisted of 22 items with 4 domains (professional support, participation, personal capacity, and safety). The modified version of this questionnaire has 23 items and the items in each area include the following: Personal capacity: items 1, 2, 4, 5, 6, 7, 21 and 22, support by professionals: items $11,13,14,15$ and 16, safety: items $3,17,18,19,20$ and 23 and participation: items 8, 9, 10 and 12. Twenty items are scored based on a 4-point Likert scale (strongly agree, most agree, most disagree, strongly disagree) and three items are scored using the Visual Analogue Scale between 0 and $100(0-40=1)(41-60=2) ;(61-80=3)$ and $(81-100=4))$. Negative experience items (experiencing pain, feeling tired, feeling scared, negative memories, and memories that trigger depressive thoughts) are scored inversely. The scores of the items in each domain are added and divided by the number of items in the same domain. The sum of the scores shows the score of the childbirth experience. The score range of this questionnaire is 23 to 92 and a higher score means a better childbirth experience. Psychometrics of this questionnaire has been confirmed by Ghanbari et al. in Iranian women (4).

The Rosenberg Self-Esteem Scale is a 10 -item scale with 5 positive and 5 negative items. This scale measures self-esteem using 4-point Likert I strongly agree to strongly disagree. Scoring in items 1, 3, 4, 7 and 10 is strongly agree $=3$ to strongly disagree $=0$ and items $2,5,6,8$ and 9 are scored in reverse (strongly agree $=0$ strongly disagree $=3$ ). Its score range is between 0 and 30 and a score of 30 indicates the highest self-esteem. The reliability of this tool has been reported in different studies from 0.82 to 0.88 $(17,18)$.

In order to check the content validity of demographic and obestetric information, and labor and delivery status questionnaires, 10 faculty members of the School of Nursing and Midwifery and midwives working in health centers were asked to express their opinions on the questionnaires and their opinions were applied at the discretion of the research team. To evaluate the reliability of the CEQ questionnaire and the Rosenberg self-esteem scale, 30 women in the postpartum stage were asked to complete the questionnaires twice at intervals of ten days. Then the intra-cluster correlation coefficient (ICC) was calculated, which a value of 0.6 and above means a good agreement between the two measurements (19). In this study, ICC value of 0.78 for CEQ and 0.73 for Rosenberg self-esteem scale showed good reliability of these questionnaires.

Participants were randomly allocated to two parallel groups, in 1:1 ratio through balanced block randomization with a block size of 4 . Prior to the study, the allocation sequence was determined by a person who was not present in the study. To conceal the allocation, the type of intervention was written in opaque closed envelopes and the envelopes were coded in the order of allocation. The questionnaires 
were also coded in order. After stating the research objectives and method for women who met the inclusion criteria, a written consent form was completed by them if they would like to participate in the study. women completed a demographic and midwifery information questionnaire before entering the study. Then, each woman received an envelope based on a predetermined sequence to be assigned to one of the intervention or control groups. In the intervention group, in addition to routine labor care, the researcher was present at the mother's bedside and was present with the mother continuously throughout the labor and up to 2 hours after delivery and provided the necessary support. This support includes emotional support (continuous presence, reassurance and praise) and information about labor progress, advice about coping techniques, comfort measures (comforting touch, massage, warm baths/showers, encouraging mobility, promoting adequate fluid intake and output) and speaking up when needed on behalf of the woman. The control group received only routine labor and postpartum care. Labor and delivery status questionnaire were completed by the midwife during study. Participants in both groups were contacted 6 weeks after delivery to complete the CEQ and Rosenberg self-esteem questionnaires. It should be noted that in this study there was no blinding (Figure. 1).

Data were analyzed using Stata-13 software. To evaluate the normality of quantitative data distribution, Shapiro-Wilk tests, histogram of data distribution as well as central tendency and dispersion were used. The results showed that all quantitative variables in this study have normal or near normal distribution. The two groups were compared in terms of demographic and obstetric variables and variables related to labor and delivery, with independent t-test or chi-square test and Fisher's exact test. One-way ANOVA/ANCOVA test was used to evaluate the differences between the two groups in terms of childbirth experience and self-esteem scores. SMD of Cohen's $d$ and $95 \% \mathrm{Cl}$ were calculated for analysis. Cohen's $\mathrm{d}$ interpretive areas were $.20-.40$ considered small, . $.50-.70$ considered moderate, $.80 \leq$ considered large (20). Analysis was performed by per protocol approach and the level of significance in all statistical tests was less than 0.05 .

\section{Results}

The mean (sd) age of women in the intervention group was 23.17 (4.68) years and in the control group was 23.09 (4.84) years. The majority of women had less than a diploma and were mostly housewives. The mean (sd) gestational age in the intervention group was slightly lower than the control group (38.71 (1.38) vs. $39.03(1.15)$ ). The results of Table 1 showed that there is no considerable difference between the two groups. 
Table 1

Demographic and obstetric information of participants

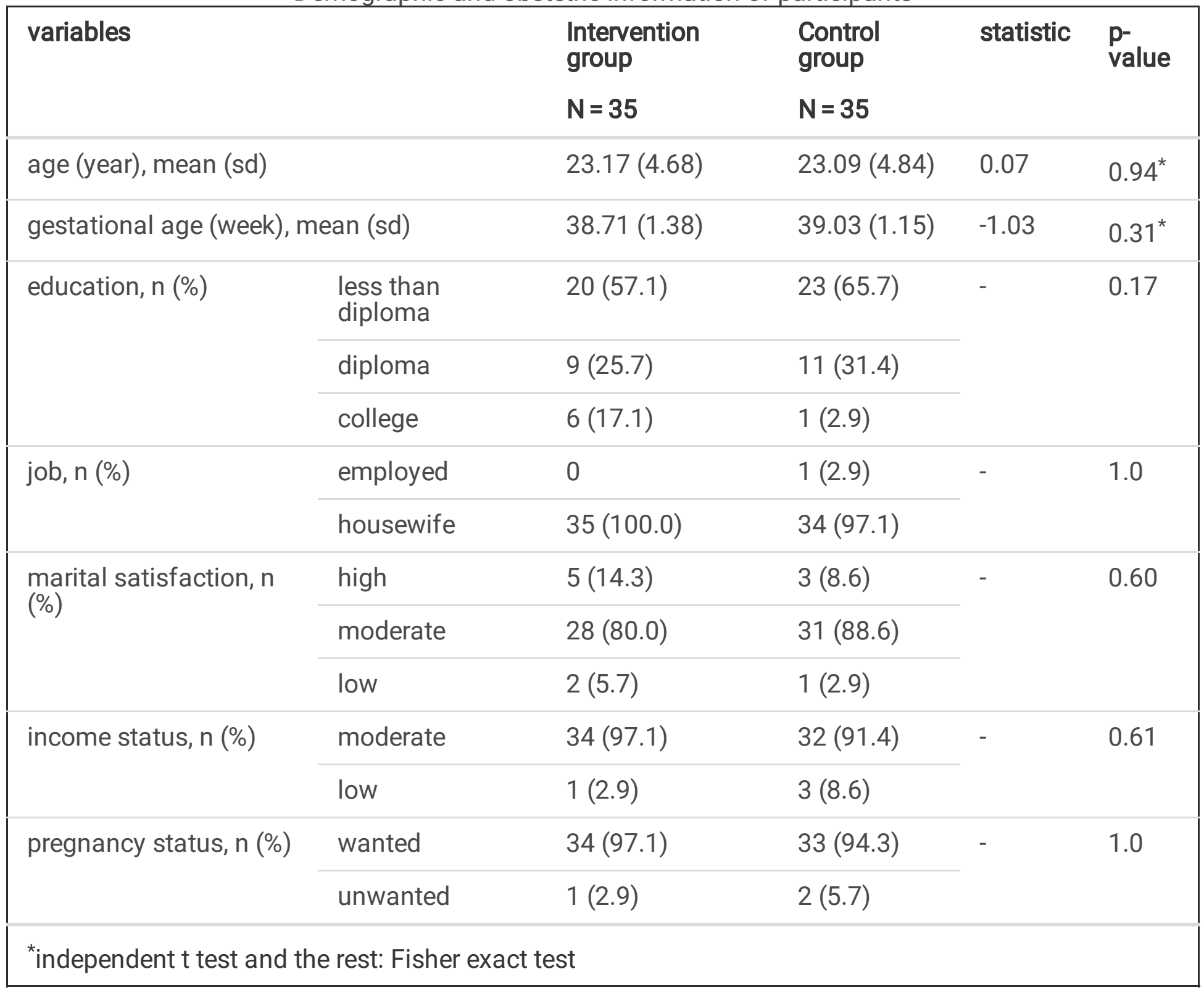

Analysis of variables related to labor and delivery showed that the mean (sd) duration of the first stage of labor in the control group was higher than the intervention group (5.08 (1.79) hours and 3.94 (1.86) hours, respectively), and this increase was also statistically significant. The mean (sd) duration of the second stage of labor was 25.76 (18.25) minutes in the control group and 15.88 (10.86) minutes in the intervention group and this difference was also statistically significant. More than two-thirds of people in labor received oxytocin in both groups, and most deliveries in the intervention and control groups were managed by a gynecology assistant $(77.1 \%$ vs. $67.6 \%)$. The rest of the information is in Table 2 . Except for the two variables of duration of the first and second stages of labor, the two groups did not considerable difference in terms of other variables. 
Table 2

Comparison of participants in terms of labor and delivery status

\begin{tabular}{|c|c|c|c|c|c|}
\hline \multicolumn{2}{|l|}{ variables $^{a}$} & $\begin{array}{l}\text { Intervention } \\
\text { group }\end{array}$ & $\begin{array}{l}\text { Control } \\
\text { group }\end{array}$ & statistic & $\begin{array}{l}\mathrm{p}- \\
\text { value }\end{array}$ \\
\hline \multicolumn{2}{|c|}{$\begin{array}{l}\text { duration of the first stages of labor (h), } \\
\text { mean (sd) }\end{array}$} & $3.94(1.86)$ & $5.08(1.79)$ & -2.57 & $0.01^{*}$ \\
\hline \multicolumn{2}{|c|}{$\begin{array}{l}\text { duration of the second stages of labor (min), } \\
\text { mean (sd) }\end{array}$} & $15.88(10.86)$ & $\begin{array}{l}25.76 \\
(18.25)\end{array}$ & -2.71 & $0.009^{*}$ \\
\hline \multicolumn{2}{|l|}{ baby's weight (g), mean (sd) } & $\begin{array}{l}3098.14 \\
(390.14)\end{array}$ & $\begin{array}{l}3060.76 \\
(309.60)\end{array}$ & 0.43 & $0.66^{*}$ \\
\hline \multirow{2}{*}{$\begin{array}{l}\text { oxytocin intake status } \\
\text { during labor, } \mathrm{n}(\%)\end{array}$} & yes & $26(74.3)$ & $23(65.7)$ & \multirow[t]{2}{*}{0.61} & \multirow[t]{2}{*}{0.43} \\
\hline & no & $9(25.7)$ & $12(34.3)$ & & \\
\hline \multirow{2}{*}{$\begin{array}{l}\text { evacuation of the placenta, } \\
\mathrm{n}(\%)\end{array}$} & spontaneous & $32(94.1)$ & $33(97.1)$ & \multirow[t]{2}{*}{-} & \multirow[t]{2}{*}{$1.0^{\star \star}$} \\
\hline & Manual & $2(5.9)$ & $1(2.9)$ & & \\
\hline \multirow[t]{2}{*}{$\begin{array}{l}\text { person managing delivery, } \mathrm{n} \\
(\%)\end{array}$} & $\begin{array}{l}\text { gynecology } \\
\text { assistant }\end{array}$ & $27(77.1)$ & $23(67.6)$ & \multirow[t]{2}{*}{1.20} & \multirow[t]{2}{*}{0.27} \\
\hline & midwife & $7(20.6)$ & $11(32.4)$ & & \\
\hline \multirow[t]{2}{*}{ sex of the baby, $n(\%)$} & girl & $18(52.9)$ & $20(58.8)$ & \multirow[t]{2}{*}{0.23} & \multirow[t]{2}{*}{0.62} \\
\hline & boy & $16(47.1)$ & $14(41.2)$ & & \\
\hline
\end{tabular}

The findings of Table 3 showed that by controlling the effect of the duration of the first and second stages of labor, the mean (sd) score of childbirth experience 6 weeks after delivery was 70.92 (7.07) in the intervention group and 59.69 (08.08). 7) in the control group and this difference was statistically significant $(p<0.001)$. The Cohen's $d$ in this outcome is $1.69(95 \% \mathrm{Cl}: 1.12,2.26)$ and indicates a very strong effect of the intervention on improving the childbirth experience. Comparison of the mean (sd) of the mother's self-esteem score showed that this rate was higher in the intervention group than the control group (21.71 (3.92) and 19.71 (3.92), respectively) and this difference was statistically significant ( $p=$ 0.05). The Cohen's d $(95 \% \mathrm{Cl}: 0.06,1.05)$ indicates the moderate effect of the intervention on mothers' self-esteem. 
Table 3

Results of multivariate analysis of childbirth experience and self-esteem scores between the two groups

\begin{tabular}{|c|c|c|c|c|c|}
\hline \multirow[t]{3}{*}{ variables } & Intervention group & Control group & \multirow[t]{3}{*}{$\begin{array}{l}\text { Cohen's d (95\% } \\
\text { Cl) }\end{array}$} & \multirow[t]{3}{*}{$\mathrm{F}^{* *}$} & \multirow[t]{3}{*}{$\begin{array}{l}\mathrm{p}- \\
\text { value }\end{array}$} \\
\hline & $N=33$ & $N=32$ & & & \\
\hline & $\begin{array}{l}\text { Adjusted mean } \\
\text { (sd) }\end{array}$ & $\begin{array}{l}\text { Adjusted mean } \\
\text { (sd) }^{*}\end{array}$ & & & \\
\hline \multirow{2}{*}{$\begin{array}{l}\text { Childbirth } \\
\text { experience }\end{array}$} & \multirow[t]{2}{*}{$70.92(7.07)$} & \multirow[t]{2}{*}{$59.69(7.08)$} & 1.69 & \multirow[t]{2}{*}{38.12} & \multirow{2}{*}{$\begin{array}{l}<.001 \\
0.00\end{array}$} \\
\hline & & & $(1.12,2.26)$ & & \\
\hline \multirow[t]{2}{*}{ self-esteem } & \multirow[t]{2}{*}{$21.71(3.92)$} & \multirow[t]{2}{*}{$19.71(3.92)$} & 0.56 & \multirow[t]{2}{*}{3.93} & \multirow[t]{2}{*}{0.05} \\
\hline & & & $(0.06,1.05)$ & & \\
\hline \multicolumn{6}{|c|}{ * adjusted for first and second stages of labor } \\
\hline \multicolumn{3}{|c|}{ **one-way ANOVA/ANCOVA } & & & \\
\hline
\end{tabular}

\section{Discussion}

One of the primary objectives of the present study was to determine the effect of continuous midwifery support in labor on the childbirth experience of nulliparous women 6 weeks postpartum. The results showed that the continuous support of the midwife and the provision of various supports to the mother have a positive effect on the delivery experience of nulliparous women. The amount of size effect showed that the effect of the intervention was very strong and considering that the $95 \%$ confidence interval includes a strong interpretive area, this result is conclusive. Namujju et al., in a qualitative study of the delivery experience two months after childbirth, concluded that the attitude, care, and support of service providers resulted in both positive and negative birth experiences. Physical and psychosocial support led to comfort, consolation, and encouragement for mothers and conversely, inadequate care, poor communication, and lack of privacy led to negative maternal childbirth experiences (12). Numerous other studies have concluded that midwife support is directly related to a positive childbirth experience $(21-23)$. Supportive care during childbirth may increase the physiological processes of childbirth as well as women's sense of control and confidence in their power and ability to give birth, and as a result, the need for intervention is reduced and women's childbirth experience is improved (6).

In some studies, the effect of education during pregnancy on women's satisfaction and childbirth experience has been studied. A study in Sweden showed that postpartum training focusing on psychoprophylactic training (breathing and relaxation techniques) in the third trimester of pregnancy had no significant effect on postpartum experience of women and their husbands (24). In another study, holding a training session to strengthen spouse support did not have a significant effect on women's childbirth experience (25). It seems that education alone cannot have a positive effect on the experience of childbirth. In our study, we were constantly present at the mother's bedside, which in addition to providing 
information, also provided various physical, psychological and emotional support to the mother. Rahimian et al. in their study showed that one-on-one midwifery care leads to higher satisfaction with childbirth (13)and in fact social support is considered as a source of comfort, encouragement and hope for mothers (12). When the mother is supported in labor, the birth hormones, which include oxytocin, endorphins, catecholamines and prolactin, are balanced with each other, and in addition to making labor pains bearable, the labor process progresses well. (26). Therefore, the presence of doula as a supportive person can play an effective role in creating maternal comfort in the delivery ward and lead to a positive childbirth experience $(26,27)$. In various studies, the effect of continuous support of midwives in labor on the short-term consequences of labor and delivery has also been investigated. In a systematic review and met-analysis study of 26 trials by Bohren et al., the authors concluded that continued support led to improved maternal and neonatal outcomes, including increased spontaneous vaginal delivery, shorter labor duration, decreased cesarean delivery, reduced use of any analgesia and regional analgesia, decreased 5-minute Apgar scores, and decreased negative childbirth experience (6).

Considering that Bohren et al. in their systematic review and meta-analysis study recommended that long-term outcomes be studied, especially in low-income countries (6), another primary objective of this study was maternal self-esteem, which was assessed 6 weeks after delivery. In our study, the continued support of midwives in labor was able to increase mothers' self-esteem compared to the control group. The amount of Cohen's d showed a moderate effect of this intervention on mothers' self-esteem, but because $95 \%$ confidence interval of this effect size covered of several interpretive areas (from ineffective to strong), the result is not conclusive and this study needs to be repeated. Hofmeyr studied in his trial the

effect of support on maternal self-esteem at 1 day, 6 weeks and one year after delivery. He concluded that the mean scores of self-esteem in the support group in the six weeks after delivery were 15.7 points higher than the control group and this difference was also statistically significant (28). Consistent with this finding, two other studies have shown that mothers' participation in decision-making during childbirth leads to increased self-esteem in them $(23,29)$.

One of the limitations of the present study was that the control of labor and delivery, and the provision of routine care in labor were performed by different midwives in different shifts. Although care is provided according to the existing protocol in the country, but the personality traits of service providers, their level of skill in managing labor and delivery, and differences in their communication skills could affect women's understanding of childbirth experience. In addition, in the present study, the conditions of the delivery ward at the time of hospitalization of each participant and the degree of crowding of delivery ward were not assessed.

\section{Conclusion}

The results of this study showed that the continuous presence of a midwife on the mother's bedside and providing the necessary support to the mother one by one in labor and up to 2 hours after delivery can lead to a better childbirth experience for the mother. Maternal self-esteem may also increase as a result of this intervention. Considering that one of the ways to reduce cesarean section is to make labor pleasant, 
it is possible to think of measures so that all mothers can benefit from the continuous support of a midwife during labor and delivery. This action in addition to encouraging mothers to have children (that declining fertility has exposed many countries to an aging population) will help countries reduce their cesarean section rates as well. The positive experience of childbirth and increasing self-esteem in mothers can affect the health of the child and family and consequently the health of society.

\section{Declarations}

Ethics approval and consent to participate: This manuscript is the result of a master's thesis student in counseling in midwifery. All interventional protocols were approved by the Vice Chancellor for Research of Hamadan University of Medical Sciences with the code 9906183918 and also the ethics committee with the code IR.UMSHA.REC.1399.474. Permission required to attend a women's hospital was obtained. The objectives of the research were explained to the participants by the researcher, and they were reassured about the confidentiality of the information and their freedom to participate or not to participate in the project. Then, informed written consent was obtained from eligible pregnant women. All methods in this manuscript were carried out in accordance with relevant guidelines and regulations.

Consent for publication: Not applicable

Availability of data and materials: The datasets used and/or analysed during the current study are available from the corresponding author on reasonable request.

Competing interests: The authors declare that they have no competing interests

Funding: The research project of this study is funded by the Vice Chancellor for Research and Technology of Hamadan University of Medical Sciences.

Authors' contribution: NS, MR and FK designed and directed the project; NS performed the intervention; FK analyzed the data; and FK wrote the article. All authors have read and approved the manuscript.

Acknowledgements: This article is the result of a master's thesis in midwifery counseling. The authors of this article would like to thank the officials of Hamadan University of Medical Sciences and Fatemieh Hospital and all the mothers who participated in this research.

\section{References}

1. Dencker A, Taft C, Bergqvist L, Lilja H, Berg M. Childbirth experience questionnaire (CEQ): development and evaluation of a multidimensional instrument. BMC Pregnancy Childbirth. 2010;10:81.

2. Zamani P, Ziaie T, Mokhtari Lakeh N, Kazemnejad Leili E. Childbirth Experience and Its Related SocioDemographic Factors in Mothers Admitted to Postpartum Ward of AL Zahra Hospital of Rasht City, (Iran). Qom Univ Med Sci J. 2019;12(11):70-8. 
3. Fenaroli V, Molgora S, Dodaro S, Svelato A, Gesi L, Molidoro G, et al. The childbirth experience: obstetric and psychological predictors in Italian primiparous women. BMC pregnancy and childbirth. 2019;19(1):1-7.

4. Ghanbari-Homayi S, Dencker A, Fardiazar Z, Jafarabadi MA, Mohammad-Alizadeh-Charandabi S, Meedya $S$, et al. Validation of the Iranian version of the childbirth experience questionnaire 2.0. BMC Pregnancy and Childbirth. 2019;19(1):465.

5. Pang MW, Leung TN, Lau TK, Hang Chung TK. Impact of first childbirth on changes in women's preference for mode of delivery: follow-up of a longitudinal observational study. Birth. 2008;35(2):121-8.

6. Bohren MA, Hofmeyr GJ, Sakala C, Fukuzawa RK, Cuthbert A. Continuous support for women during childbirth. The Cochrane database of systematic reviews. 2017;7(7):Cd003766.

7. World Health Organization. Standards for improving quality of maternal and newborn care in health facilities. 2016.

8. Oosthuizen SJ, Bergh A-M, Pattinson RC, Grimbeek J. It does matter where you come from: mothers' experiences of childbirth in midwife obstetric units, Tshwane, South Africa. Reproductive health. 2017;14(1):151.

9. Darvishi M, Ranjbaran M, Jourabchi Z, Jafarimanesh H. Effect of the presence of midwife and nonmidwife companion on labor pain, duration of delivery, and amount of oxytocin drops in primiparous women: a clinical trial study. The Iranian Journal of Obstetrics, Gynecology and Infertility. 2019;22(4):63-72.

10. Lunda P, Minnie CS, Benadé P. Women's experiences of continuous support during childbirth: a metasynthesis. BMC pregnancy and childbirth. 2018;18(1):167.

11. Hodnett ED. Caregiver support for women during childbirth. The Cochrane database of systematic reviews. 2000(2):Cd000199.

12. Namujju J, Muhindo R, Mselle LT, Waiswa P, Nankumbi J, Muwanguzi P. Childbirth experiences and their derived meaning: a qualitative study among postnatal mothers in Mbale regional referral hospital, Uganda. Reprod Health. 2018;15(1):183.

13. Rahimiyan MN, Rahnavard T, Lari MZ. Effect of the one to one Midwifery Care During Labor on Modes of Delivery and Duration of Labor and Increase Satisfaction with Childbirth. Biosci Biotech Res Asia. 2015;12:839-45.

14. Kashanian M, Javadi F, Haghighi MM. Effect of continuous support during labor on duration of labor and rate of cesarean delivery. International Journal of Gynecology \& Obstetrics. 2010;109(3):198200.

15. Safarzadeh A, Beigi M, Salehian T, Khojasteh F, Burayri T, Navabirigi S. Effect of Doula support on labour pain and outcomes in Primiparous women in Zahedan, southeastern Iran: A randomized controlled trial. J Pain Relief. 2012;1(5):2167-0846.1000112.

16. Lathrop A, Bonsack CF, Haas DM. Women's experiences with water birth: A matched groups prospective study. Birth. 2018;45(4):416-23.

Page 11/13 
17. Babington LM, Malone L, Kelley BR. Perceived social support, self esteem, and pregnancy status among Dominican adolescents. Applied nursing research: ANR. 2015;28(2):121-6.

18. Dolatian M, Mirabzadeh A, Setareh Forouzan A, Sajjadi H, Alavi Majd H, Moafi F, et al. Correlation between self-esteem and perceived stress in pregnancy and ways to coping with stress. Pajoohande. 2013;18(3):148-55.

19. Cicchetti DV, Sparrow SA. Developing criteria for establishing interrater reliability of specific items: applications to assessment of adaptive behavior. American journal of mental deficiency. 1981.

20. Cohen J. Statistical power analysis for the social sciences. 1988.

21. Corbett CA, Callister LC, Gettys JP, Hickman JRJTJop, nursing n. The Meaning of Giving Birth. 2017;31(3):207-15.

22. Halldorsdottir S, Karlsdottir SIJM. Journeying through labour and delivery: perceptions of women who have given birth. 1996;12(2):48-61.

23. Murray L, Windsor C, Parker E, Tewfik OJHcfwi. The experiences of African women giving birth in Brisbane, Australia. 2010;31(5):458-72.

24. Bergström $\mathrm{M}$, Kieler $\mathrm{H}$, Waldenström $\mathrm{U}$. Effects of natural childbirth preparation versus standard antenatal education on epidural rates, experience of childbirth and parental stress in mothers and fathers: a randomised controlled multicentre trial. BJOG: an international journal of obstetrics and gynaecology. 2009;116(9):1167-76.

25. Eom SY, Kim ES, Kim HJ, Bang YO, Chun NJJoKAoN. Effects of a one session spouse-support enhancement childbirth education on childbirth self-efficacy and perception of childbirth experience in women and their husbands. 2012;42(4):599-607.

26. Simkin P, Hanson L, Ancheta R. The Labor Progress Handbook: Early Interventions to Prevent and Treat Dystocia: Wiley; 2017.

27. Koumouitzes-Douvia J, Carr CAJTJope. Women's perceptions of their doula support. 2006;15(4):34.

28. Hofmeyr GJ, Nikodem VC, Wolman WL, Chalmers BE, Kramer T. Companionship to modify the clinical birth environment: effects on progress and perceptions of labour, and breastfeeding. $\% \mathrm{~J}$ BJOG: An International Journal of Obstetrics. 1991;98(8):756-64.

29. Simkin PJB. Just another day in a woman's life? Women's long-term perceptions of their first birth experience. Part I. 1991;18(4):203-10.

\section{Figures}




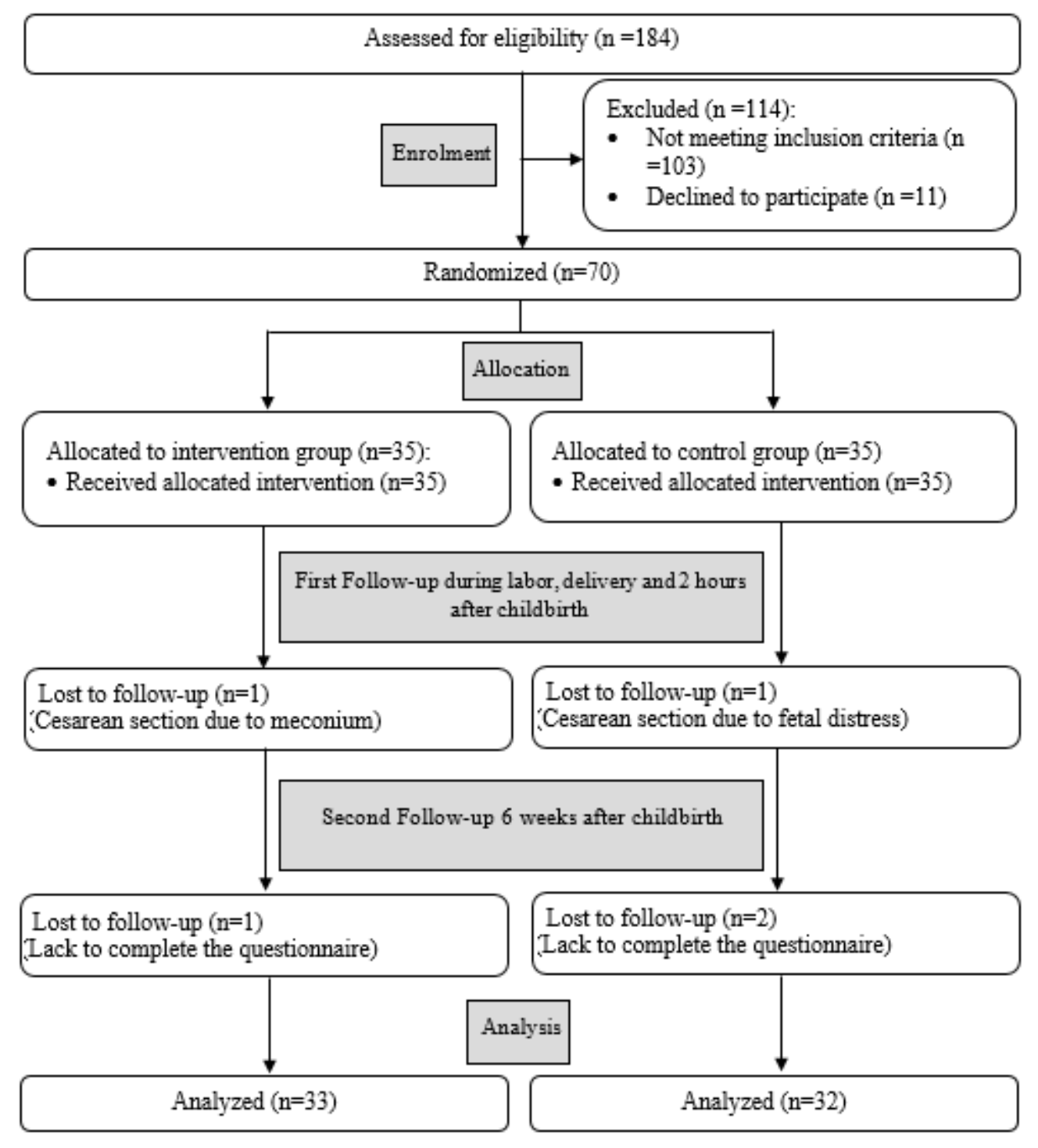

Figure 1

flowchart of participation 